\title{
Development and design of fitness exercise prescription system based on cloud storage
}

\author{
Ying Li \\ Pingxiang University, Jiangxi 337055, China \\ 359020645@qq.com
}

Key words: cloud storage; exercise prescription; development; design

\begin{abstract}
. with the continuous improvement of people's living standard, people pay more and more attention to their own health problems. The information system for the system of exercise prescription has not attracted the attention of many people, how to make use of the advantages of cloud storage, to share the exercise prescription data, helps people to timely feedback and fitness fitness, for future wearable devices provide the required monitoring data, for people to provide more quality services, improve people's physical quality.
\end{abstract}

\section{Cloud storage concept}

Cloud computing is a distributed computing, parallel computing, utility computing, network storage products and other traditional computer and network technology mixed, include infrastructure as a service (Iaas), platform as a service (PaaS), software as a service (SaaS) and other three kinds of Internet service. It completes the computation tasks through distribution the form of a large number of computers in the resource pool, at the same time so that all applications can according to need to obtain computing power, storage space and software services

Cloud storage is an extension of the concept of cloud computing. Cloud computing is a computer program through the Internet will require complex operations into several smaller subroutines, after complete distributed computing through the computer network analysis results will be feedback to the user in this way, the demand for cloud computing can be completed in a short time to calculate hundreds of millions this time, computing power can even reach and exceed the level of super computer. And cloud storage is cloud computing through the above method, through a large number of storage devices in the network, the user data stored therein.

\section{The data model for the development of exercise prescription}

In general ADO.NET has two characteristics: one is to connect different data sources to support a variety of.NET data access procedures set.ADO.NET for the data warehouse, data warehouse is a description of the corresponding instructions, finally get some results from the database, followed by the data provider is responsible for implementation of controlled two. Microsoft SQL Server is a local method or other types of tabular data stream provides ADO.NET; for ADO.NET, the new Data Reader class can make the ability to obtain data flow is greatly improved. 


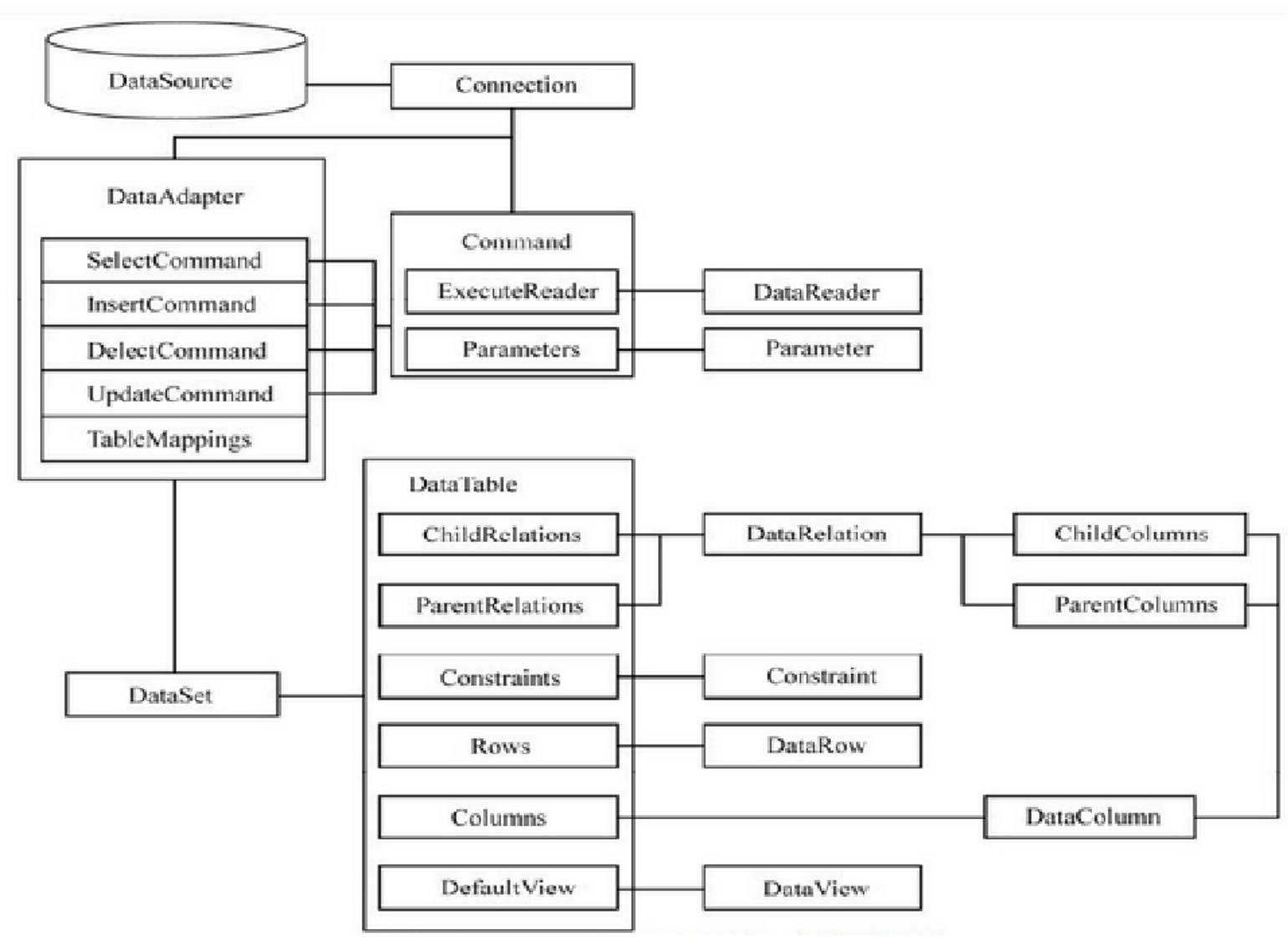

Chart 1 ADO.NET data model

\section{The design of the work flow of the exercise prescription system}

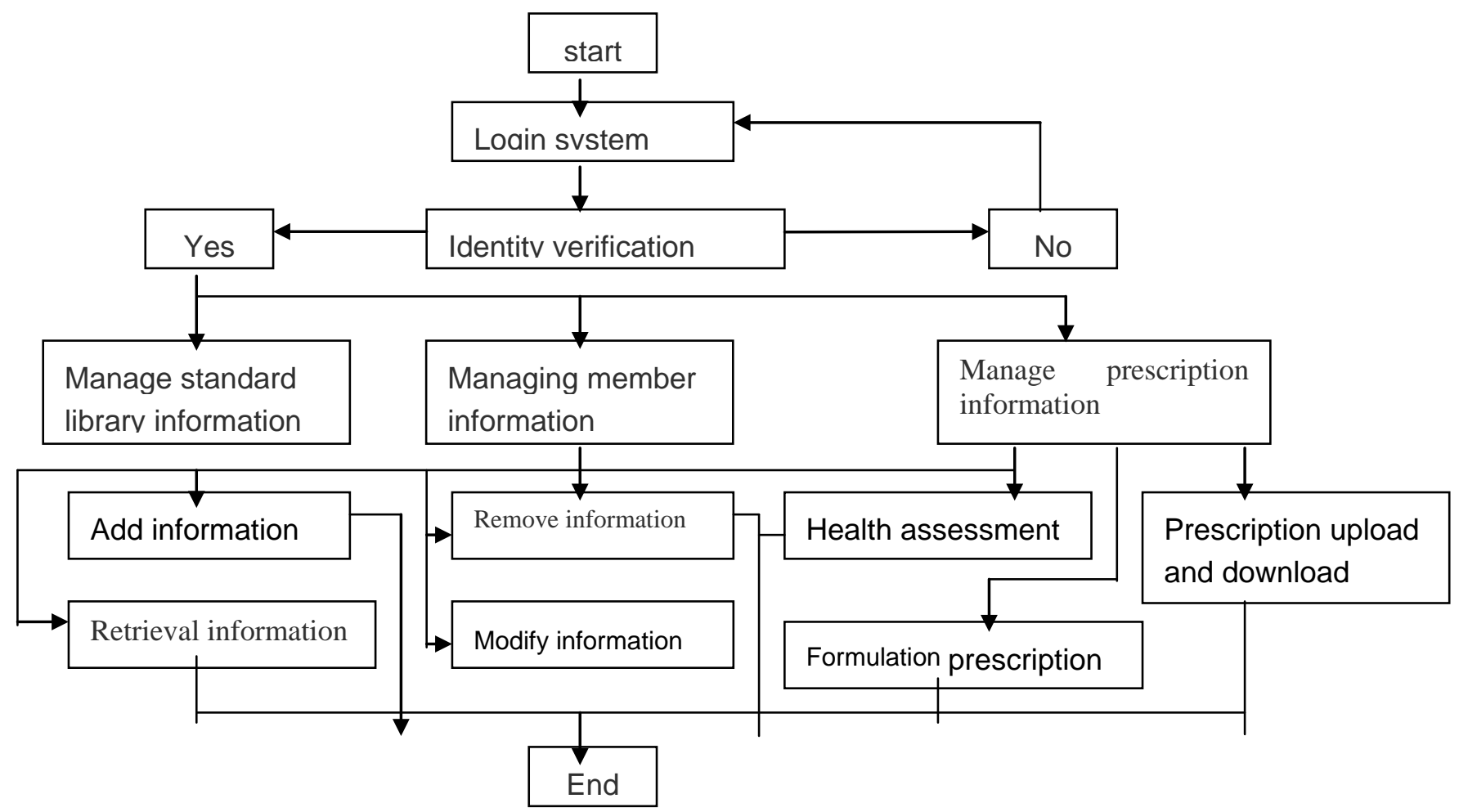

Chart 2 The exercise prescription system workflow 
3.1 User authentication: make comparison of user input via authentication information and confirm their own after the system database and original data storage, if the information is consistent well into the system, and notify the system authentication failure, return re certification.

3.2 Add exercise prescription data function: the whole exercise prescription system needs to input a variety of data, including administrator information, membership information, sports test standard library information, questionnaire information, a variety of sports test information.

3.3 Remove the exercise prescription system data function: the entire exercise prescription system need to remove a variety of data, including the administrator information, membership information, exercise test library information, questionnaire information, a variety of sports test information, health assessment and exercise prescription information. In the delete operation at the same time, the system will ensure that the user cannot delete the abnormal flow the empty record sound, while ensuring that the user can delete cascade, so as to ensure the consistency of the database.

3.4 Records in the database information retrieval function: the system is all of the information query, the query module provided by the system, users can complete the function of fuzzy query. Users enter through a unified query query module, the system will automatically match the page according to the user query, query the user the freedom to choose the logic combination, finally the user desired result output.

3.5 Modify the database of exercise prescription records: the entire exercise prescription system of all the data, including the administrator member information, exercise testing, questionnaire and other direct input of information can be directly modified.

3.6 Questionnaire survey: the history of the user, lifestyle, disease signs, PAQ-Q questionnaire was completed.

3.7 Exercise tests: SCL90 psychological test for the user, physiological and biochemical index, body shape index test, exercise test test total four groups of different aspects of the test, each test includes at least one refinement sub test, test each can do in different time, need not be completed at the same time however, each test required to complete the refinement test in a test, test after the completion of each test and refinement can be edited or deleted, can also be used in the next new test to replace, and automatically record the last test time. Users can query to view their own tests.

3.8 Health assessment: the body shape index system will be completed for the user test, exercise test, SCL90 psychological test, four aspects of physiological and biochemical tests to assess each user to select at least one test, the system will be according to the standard letter body index in the standard library information for the selected test automatic health assessment.

3.9 Prescription generation: according to the generated health assessment information, the system can be generated according to the prescription of exercise prescription for the user to customize the prescription information, the user can print the final formulation of the exercise prescription.

3.10 Prescription upload and download: through the use of Microsoft's cloud service platform SkyDrive, the completion of the exercise prescription of the XML template upload, through the mobile terminal app obtained from the SkyDrive XML file and display correct exercise prescription for users.

\section{Fitness exercise prescription module design}

From the business needs analysis of sports prescription system is mainly divided into three modules: member and library information management module, health testing and evaluation and exercise prescription generation module, exercise prescription upload and download module.

\subsection{Member and library information management module}

4.1.1 Administrator settings: for administrator privileges to set up, while adding a new coach to become an administrator, delete leave to teach and to view the administrator's online situation, but also to modify the administrator privileges. 
4.1.2 Member information management: membership of some of the basic information management, including the name, gender, occupation, education, etc., to determine the user's user groups.

4.1.3 The standard library maintenance: maintenance of the questionnaire, some physical standards required by the test (including crud), for health standards improve constantly changing, to provide users with the most advanced psychological test, so as to achieve the scalability of the exercise prescription.

4.1.4 Other library maintenance: the maintenance of some commonly used recommendations and the user community library, including user groups and other categories.

\subsection{Health assessment and exercise prescription generation module}

4.2.1 Health Questionnaire: contains the history investigation on the user's way of life assessment, disease signs survey questionnaire (PAQ-Q PAQ-Q questionnaire is a survey questionnaire, the sports medicine recommended that for moderate intensity activity, should be able to pass the minimum requirement, or the need for medical examination).

4.2.2 Exercise test: contains physiological and biochemical test, exercise test, SCL90 psychological test, body shape index test. Each test and the need for 2-14 in different sub project movement testing or refinement of physiological indicators.

4.2.3 Health assessment: the generation, search, and view of assessment.

4.2.4 Exercise prescription: include the generation of exercise prescription, view, search.

\subsection{Upload and download module of exercise prescription}

4.3.1 Exercise prescription upload: from the PC side of the exercise prescription object is to be uploaded to the XML file upload.

4.3.2 Download the exercise prescription: download the XML file from the cloud to the exercise prescription, and provide the function of anti serialization, the final resolution is displayed in the mobile terminal.

\section{Exercise prescription system function design}

In order to realize the goal of the system, must design all program and database of the system, and the structural design of the program and database. Usually, each sub module of the system is responsible for the completion of a single function. Each module concrete structure: by calling the lower module top module the realization of the whole system. Each of the lower module calls lower level modules, the bottom of the module to realize the function of the system, thus completing a the corresponding sub function module. 


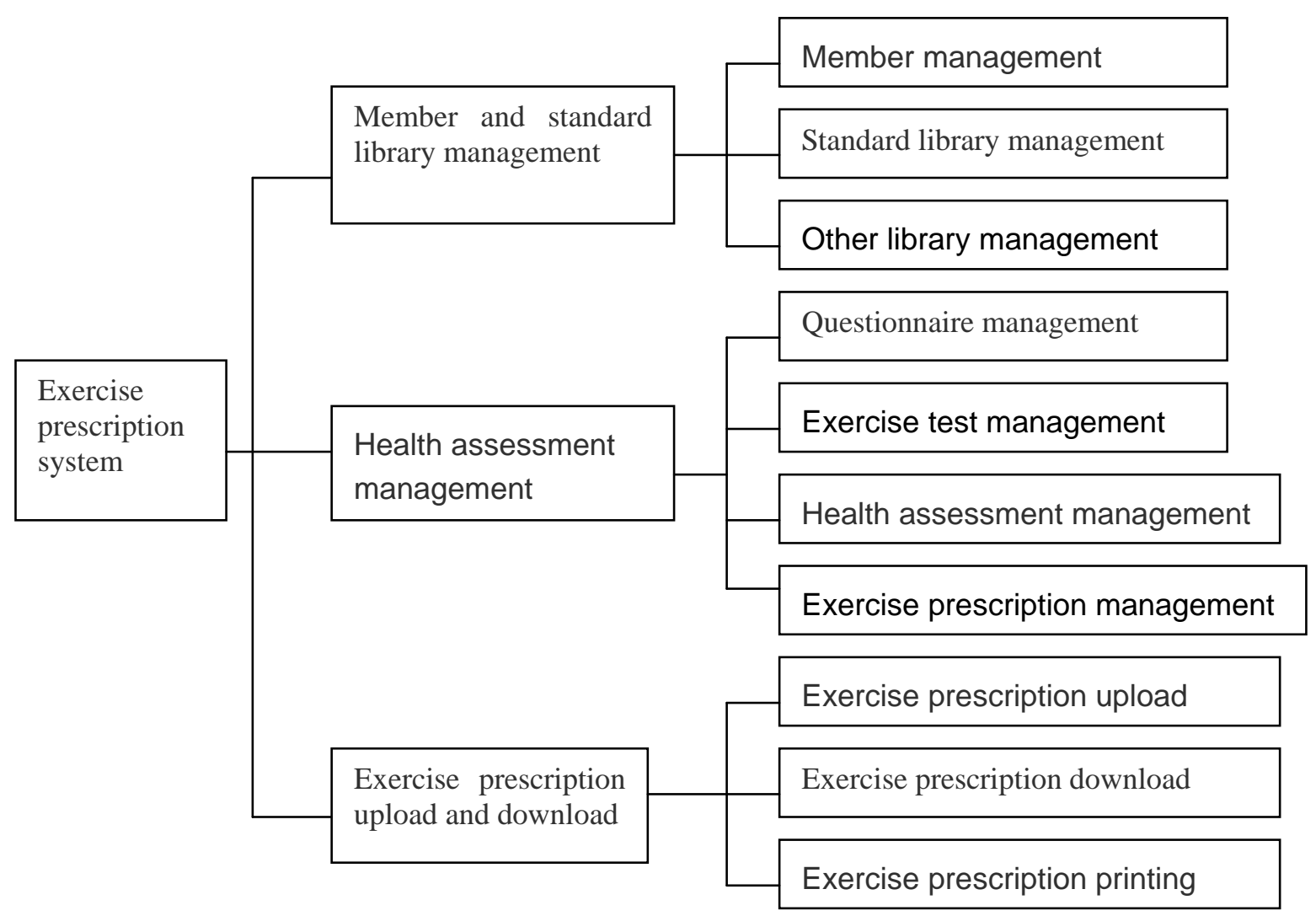

\section{Chart 3 Exercise prescription system function module decomposition}

According to the system level diagram shown in Figure 4.2, the exercise prescription system from top to bottom should be divided into three layers. The first layer of user authentication, user login information to determine whether the user has permission to log on. The second layer is the management system function module mainly includes the member with the standard library management, health management, upload and download exercise prescription. The three module is the main function of exercise prescription system. The third layer includes membership management, library management, questionnaire management, exercise test management, health assessment, prescription upload and download module

Every single business module are in accordance with the hierarchical structure as mentioned above, according to the standards of a complete business from top to bottom including presentation layer, business layer, data access layer, persistence layer. The persistence layer is mainly related to database operations, which provide the basic data for business support system; data access layer the main is to integrate the data persistence layer, and all data will be mapped to the corresponding class; the presentation layer is the main user control logic on the presentation layer, according to the user request triggers the corresponding event, the business logic will finally return the results to the user request triggering event, and appear in the presentation layer.

\section{Reference}

[1] Jun-hua Xu, Yang Jingyi. Sports prescription [M]. Beijing: Higher Education Press, 2005

[2] Chuan-hui Yang, large-scale distributed storage system: the principle of analysis and architecture of the actual combat [M]. Machinery Industry Press; first edition, 2013 